\title{
Identifying core components and indicators of successful transitions from child to adult mental health services: a scoping review
}

\author{
Kristin Cleverley ${ }^{1,2} \cdot$ Emily Rowland $^{3}\left(\mathbb{C}^{\circ} \cdot\right.$ Kathryn Bennett $^{4,5} \cdot$ Lianne Jeffs $^{6,7} \cdot$ Dana Gore $^{6}$
}

Received: 6 January 2018 / Accepted: 11 August 2018 / Published online: 8 October 2018

(c) The Author(s) 2018

\begin{abstract}
The aim of this scoping review was to identify the core components of interventions that facilitate successful transition from child and adolescent mental health services to adult mental health services. In the absence of rigorous evaluations of transition program effectiveness for transitioning youth with mental health care needs, these core components can contribute to informed decisions about promising program and intervention strategies. This review examined data from 87 peer-reviewed and non-academic documents to determine the characteristics that support the transition process and to identify opportunities for system and program improvement. Data were extracted and synthesized using a descriptive analytic framework. A major finding of this review is a significant lack of measurable indicators in the academic and gray literature. This review did identify 26 core components organized within the framework of the six core elements of healthcare transitions. Policy makers, practitioners, and administrators can use the core components to guide decisions about transition program and intervention content. Confirmation of the impact of these core program components on youth outcomes awaits the conduct of rigorous randomized trials. Future research also needs to explicitly focus on the development of indicators to evaluate transition programs and interventions.
\end{abstract}

Keywords Transition $\cdot$ Mental health $\cdot$ Youth $\cdot$ Scoping review

Kristin Cleverley

k.cleverley@utoronto.ca

1 CAMH Chair in Mental Health Nursing Research, Lawrence S. Bloomberg Faculty of Nursing and Department of Psychiatry, University of Toronto, 155 College Street, Suite 130, Toronto, ON M5T 1P8, Canada

2 Centre for Addiction and Mental Health, Toronto, Canada

3 Dalla Lana School of Public Health, Social and Behavioural Health Sciences, University of Toronto, Toronto, ON, Canada

4 Department of Health Research Methods, Evidence and Impact (Formerly Clinical Epidemiology and Biostatistics), McMaster University, Hamilton, Canada

5 Offord Centre for Child Studies, McMaster University, 1280 Main St W, Hamilton, ON L8S 4L8, Canada

6 Lawrence S. Bloomberg Faculty of Nursing, University of Toronto, Toronto, Canada

7 Keenan Research Centre of the Li Ka Shing Knowledge Institute, St. Michael's Hospital, 30 Bond Street, Room 720, Toronto, ON M5B 1W8, Canada

\section{Introduction}

Optimal mental healthcare is crucial throughout the life course, yet the transition from child and adolescent mental health services (CAMHS) to adult mental health services (AMHS) has been recognized as a uniquely problematic international health systems hurdle [1-9]. This common demarcation in mental health services that occurs at age 18, when youth must transition from CAMHS to AMHS, creates the risk of transition-related discontinuity of care. Consequently, these youth are vulnerable to decrements in their mental health and functioning [10-13].

In mental healthcare, transition is distinct from transfer of care. Transfer is the termination of CAMHS and re-establishment of care in AMHS [14]; transition aims to ensure continuity of care through a planned, personalized, health care process that addresses therapeutic and developmental needs [15]. Continuity of care is defined by: (a) how a patient experiences care over time and (b) how care is received by a patient across the episodes of care [16]. Continuity of care must be coherent and linked as a result of good information flow, interpersonal and 
readiness skills of the youth, and coordination of care [16]. Successful continuity of care is contingent upon optimal transition from CAMHS to AMHS. However, the transition process is often a negative one for youth and their caregivers $[17,18]$ and as many as six of ten youth stop accessing mental health services [13, 19]. Even with this recognition, there is a paucity of research evaluating interventions that improve the transition process for youth as they move from child to adult services [20].

A recent scoping review of interventions to support CAMHS-AMHS transitions revealed that very few interventions have been developed and evaluated, and that none were standardized [21]. The lack of evaluated programs and interventions may be due in part to a lack of consensus on what the core components and corresponding quality and process indicators of successful transitions are. Core components are defined as the most essential and indispensable components of an intervention or program and should identify how the traits are developed, if they are replicable and the environments in which they can be observed [22]. Indicators are quantitative measures that can be used to monitor and evaluate the quality of interventions and outcomes of CAMHS-AMHS transition programs [23]. This gap in standardized and evaluated interventions has remained unchanged in recent years, even though experts identified understanding "how to measure a successful transition" or "evaluate a transition program" as two key research priorities more than 5 years ago [24].

It is important to address these gaps as not only are clinicians, researchers, and policy demanding greater understanding of the mental healthcare transition process but youth and their families are requesting improvements to continuity of mental health care [1, 3, 25-27]. Hence, this scoping review explores the literature on mental health transitions to identify core components and indicators that facilitate CAMHS-AMHS transition programs and interventions.

\section{Methods}

This scoping review applied the six-stage scoping review framework of Arksey and O'Malley [28, 29] as outlined in the study protocol [30].

\section{Stage 1: research questions}

1. How has a 'successful transition' from CAMHS to AMHS been defined and operationalized?

2. What core components and indicators have been used to define and evaluate CAMHS-AMHS transition programs and interventions?

\section{Stage 2: search strategy}

The search strategy was developed by an experienced research librarian. For the published literature, the following databases were included: Medline, Embase, PsycINFO, Cochrane Database of Systematic Reviews (all OVID interface), CINHAL (EBSCO), Applied Social Science Index and Abstracts (ProQuest), Sociological Abstracts (ProQuest) and the Campbell Library. In addition, the gray (unpublished) literature search included the following databases: Google Advanced and the Canadian Agency for Drugs and Technologies in Health search tool, 'Grey Matters' [31]. The detailed search strategy is listed in an Appendix in the published protocol [30]. In summary, we used a combination of controlled vocabulary and text words for the key concepts of transition and child, adolescent, and adult mental health. The gray literature search used similar search terms and targeted child/adolescent mental healthcare providers, agencies and support services. The search was limited to English language studies published between 1980 and 2016. The reference lists of relevant articles were searched by hand to identify articles and documents that were not generated in the database search.

\section{Stage 3: study selection}

\section{Title and abstract review}

Study selection began with a review of the titles and abstracts based on inclusion criteria. All research studies (experimental, quasi-experimental, observational) and non-research studies (guidelines, narrative reviews, policy documents) examining the transition from CAMHS to AMHS were included. Title and abstract review was not completed on the gray literature given the length of these documents and the lack of abstracts. Inclusion criteria consisted of research and non-research publications that examined the transition from CAMHS to AMHS. Studies from acute care, primary care and community services were included. Studies and documents that focused primarily on the developmental disorders, autism, and associated intellectual and developmental disabilities (IDD) were excluded given the primary focus of the review on mental disorders as the diagnosis.

Two researchers (KC and a graduate student) pilot tested the eligibility criteria using a random sample of 25 articles. Using the Cohen $k$ coefficient, the inter-observer agreement of the pilot test was kappa 0.88 (95\% CI $0.66-1.00)$, which is considered almost complete agreement [32]. Using Cochrane review software (Covidence), 
the reviewers then independently screened each retrieved title and abstract for eligibility using the inclusion criteria.

\section{Full-text review}

The full text of the remaining peer-reviewed articles and all gray literature documents were then screened by $\mathrm{KC}$ and a graduate student for eligibility. In the event of a conflict, a third reviewer (ER) was consulted for resolution.

\section{Stage 4: charting the data}

Data were extracted from all eligible articles and entered into Microsoft Excel by the second author (ER). As detailed in the protocol [30], data extracted included: publication type, study aim/objectives, definition of transition, definition of transition success, description of transition intervention or program, description of core components of transition, name and description of indicator, indicator type (process, outcome), methods for measurement, evidence to support the indicator, and results (if applicable). The first author (KC) extracted data from five randomly selected articles to ensure reliability of the content entered in each category.

Data were then organized and analyzed using a directed content analysis approach [33], referring back to the research questions as a guide [29]. Duplicate components or characteristics were removed, and categories with the same meanings collapsed. This iterative process facilitated the identification of how transition was being defined in the field and how larger themes would be determined. Some extracted data fit into more than one category. The research team reviewed the data and categories as a group to achieve consensus. A third reviewer with a Master's level education was included in the data extraction process to ensure validity and reduce bias.

\section{Stage 5: collating, summarizing and reporting results}

The team organized the content according to the broad categories described in stage four to reflect the patterns and unique explanations. To answer the first research question, definitions of transition success were collated, and then the number of times a particular definition was utilized across documents was recorded. The data extracted on the core components and indicators of CAMHS-AMHS transition (question 2) were summarized using a directed content analysis framework [33]. This entailed reviewing the data and identifying which stage of the transition process it described or informed. To ensure reliability and agreement of the content being extracted, the two researchers (KC and ER) met frequently to review each stage of the analysis and the data captured in each category.

\section{Results}

\section{Characteristics of documents}

Initial database searches yielded 1212 articles for screening (see flow chart, Fig. 1). Following title and abstract screening, 156 peer-reviewed documents met the study inclusion criteria and were retrieved for full-text review. The 39 documents from the gray literature search were then included for a total of 195 documents for full-text review. After full-text review, 86 documents were eligible: 56 peer-reviewed journal publications and 30 gray literature documents. All articles were published between 1990 and 2016 with the majority (81\%) being published since 2010 . In total, 55 of the articles $(65 \%)$ were published studies from the UK, 11 from the US, 9 from Canada, 3 from Italy, 2 from Ireland, 2 from Sweden, 2 from Australia, and 1 from Scotland. The peer-reviewed publications included qualitative studies $(n=14)$, quantitative studies $(n=17)$, and reviews $(n=9)$. The remaining non-original research consisted of discussions, theoretical perspectives $(n=8)$ and commentaries $(n=16)$. The gray literature mostly consisted of program reports $(n=11)$ or guidelines aimed at policymakers and health care providers $(n=8)$. The remainder were briefings $(n=1)$ and position statements $(n=2)$. While most $(n=67)$ papers and documents focused broadly on mental health conditions, few focused on specific mental health diagnosis such as attention deficit/hyperactivity disorders $(n=15)$ and eating disorders $(n=4)$.

\section{Question 1: operationalization of child to adult mental health transition success}

No standard definition of CAMHS-AMHS transition success was applied consistently across the literature, although there was consensus on some aspects. For example, three articles point to transition being a process and not a single event in time [14, 34, 35]. There was consistent use of two definitions of mental health transitions throughout the documents, with 22 papers citing the same two references, one broadly related to health transitions in general [15] and the other specific to CAMHS-AMHS transitions [13]. The first is from Blum et al. [15], who conceptualize a transition as "the purposeful, planned movement of adolescents and young adults with chronic medical conditions from child-centered to adult-oriented health care systems". Blum's definition was referred in nine of the reviewed articles, yet it is not specific to transitions in mental health care $[3,7,13,14,36-40]$.The second is from Singh et al. [13], who build on the definition 


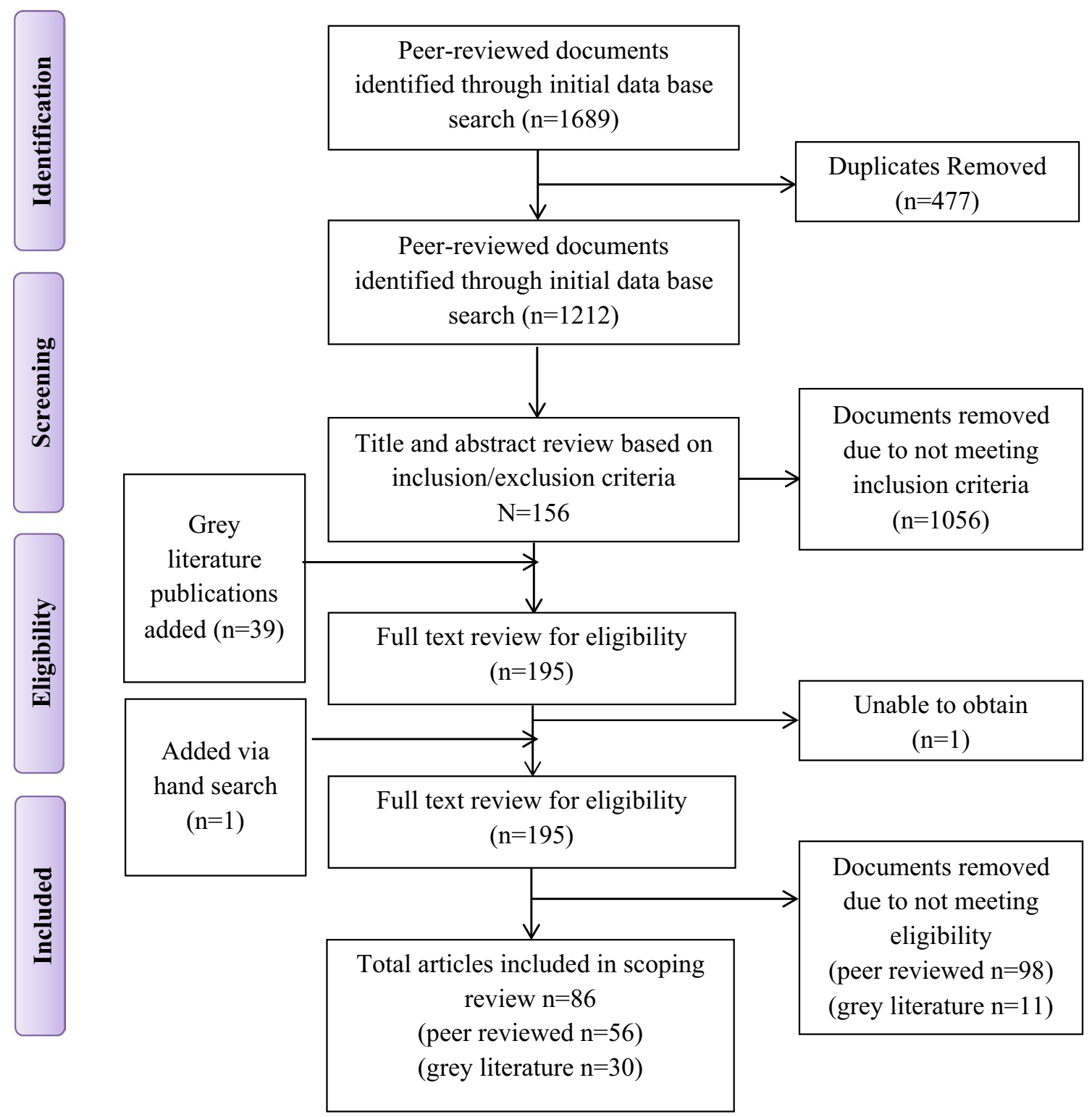

Fig. 1 Flow chart of search results

from Blum et al. [15], adding four specific criteria for determining whether transitions in care were optimal or successful. Further, unlike Blum et al. [15], these criteria are specific to transitions from CAMHS to AMHS. These criteria were used as definitions and key elements for transition success in 15 articles reviewed [5, 11, 13, 14, 20, 39, 41-49]. The criteria include: (a) information transfer: evidence that a referral letter, summary of CAMHS care, or CAMHS case notes were transferred to AMHS along with a contemporaneous risk assessment; (b) period of parallel care: a period of joint working between CAMHS and AMHS during transition; (c) transition planning: at least one meeting involving the service user and/or carer and a key professional from both CAMHS and AMHS prior to transfer of care; and (d) continuity of care: either engaged with AMHS 3 months post-transition or appropriately discharged by AMHS following transition.

\section{Question 2: core components and indicators}

None of the publications reviewed by the research team described quantifiable indicators as defined by Mainz [23]. However, the papers reviewed did describe and recommend components of interventions or programs that promoted successful transitions from child to adult mental health services. One particular framework found in the reviewed literature, Got Transition [12], identified six core elements and corresponding core components of transitions from child to adult 
physical health services. These six core elements (transition policy, transition tracking and monitoring, transition readiness, transition planning, transfer of care and transfer completion) are designed to guide interventions and programs for child to adult transition in physical health care. The research team used these six core elements as the overarching themes to organize our scoping review's core component findings $[12,50]$. However, the components in this scoping review are specific to CAMHS-AMHS transitions. Table 1 presents the core elements, core components, and corresponding references included in this review.

\section{Core element 1: transition policy}

Transition policies discussed in the literature address age, service, and geographic boundaries. The components identified in this section describe how organizations and clinical programs ought to enhance their organizational readiness for transitions of their clients. This could be done through development and implementation of distinct policies, protocols, and staff training on the transition from CAMHS to AMHS. This review identified several documents that relate to policy and organizational issues and that examine the role of organizational readiness in the child to adult mental health service transition process (see Table 1). Hoffman et al. [1] state that youth, families and health care providers in the mental health system are governed by the policies that influence the clinical operation of programs and services, such as policies that dictate age and geographic boundaries. They argue that new policies are needed to enhance collaboration and ensure cross-boundary services are working together and pooling resources to provide optimal support for providers and families. These policies should be developed with an understanding of how clinicians and organizations operate programs and services, as well as how they will affect service users (i.e. youth and their caregivers) [51]. As such, an important aspect of developing transition policy is the inclusion of youth and their caregivers in its development $[1,3,13,35,46,52-60]$.

Recommendations were made about specific actions organizations can implement to facilitate successful transitions, including ensuring tailored education and staff training about transitions that is coordinated across both CAMHS and AMHS $[1,3,4,13,20,34,35,38,39,42-44,49,54,57$, 60-80]. This would include education on the unique needs of transition-aged youth [44, 68-70, 74]-specific diagnoses such as ADHD [42, 80], services in CAMHS, AMHS and the voluntary sector $[67,75,77]$, and shared values and practices across CAMHS and AMHS [43, 69, 71, 75]. Several documents recommended that specific roles and responsibilities of all individuals involved in the transition process need to be determined and agreed upon $[3,13,20,34,35$, $56,59,60,71,76,81-83]$.
The use of integrated care pathways (ICPs) was also suggested as a way to provide a clear overview of the organization's approach to the transition process $[1,3,20,44,46$, 48, 51-53, 57, 59-61, 66, 67, 71, 73, 76, 80, 84-91]. ICPs are "structured, multidisciplinary care plans which detail essential steps in the care of patients with a specific clinical problem" [92]. They can increase consistency between programs and services, and formalize a currently informal process $[49,73]$. An integral part of the ICP is a transition protocol that includes specific standards for communication, information sharing and record-keeping between CAMHS and AMHS [1, 3, 5, 13, 35, 42, 44, 48, 51, 52, 55-58, 60, 62, $63,66,67,70-76,79,80,85,86,89,91,93-100]$, as well as alternative planning for youth who are not able to connect with AMHS upon discharge from CAMHS or who withdraw temporarily from services $[54,56,60,75,88]$.

Twenty-six articles identified the importance of using developmental readiness as a catalyst for transition rather than a chronological age (e.g. age 18) $[4,5,13,26,40,44$, $45,50,52,56,59,60,62,63,68,73-76,80,81,83,89,90$, 98, 101]. For example, Reale et al. [5] stated that developmentally defined boundaries rather than boundaries based on a fixed age will facilitate transition as a process rather than an event in time. The need for policies related to services and programs emphasized that care should be provided in accordance with the developmental needs of the youth. Establishing what is developmentally appropriate for youth ensures that their needs are being met and that transition is not simply an administrative process [48] prompted by age milestones [36, 48, 68, 102].

Last, 16 documents acknowledged the significance of assessing the quality of transition programs through a formal evaluation $[34,52,54,56,59,62,63,69-71,75,76,79,90$, $98,103]$. However, no documents provided specific process or outcome indicators by which this could be achieved.

\section{Core element 2: tracking and monitoring}

Twelve papers described the need for criteria to assist clinicians and administrators in identifying youth who will be transitioning out of CAMHS prior to the confirmed transition date or deadline $[26,52,54,63,64,76,78,80,85$, 103-105]. Youth frequently transition between services and out of care. As a result, there should be a formal process for tracking and managing these transitions. McManus et al. recommend developing specific transition criteria and a process to track and monitor individuals at all stages of the transition [106]. Six sources recommended that this process be facilitated by a flow sheet or log book [50, 54, 61, 76, 104, 106]. Additionally, the Care Quality Commission [105] of the UK recommended monitoring as an effective way to address wait 


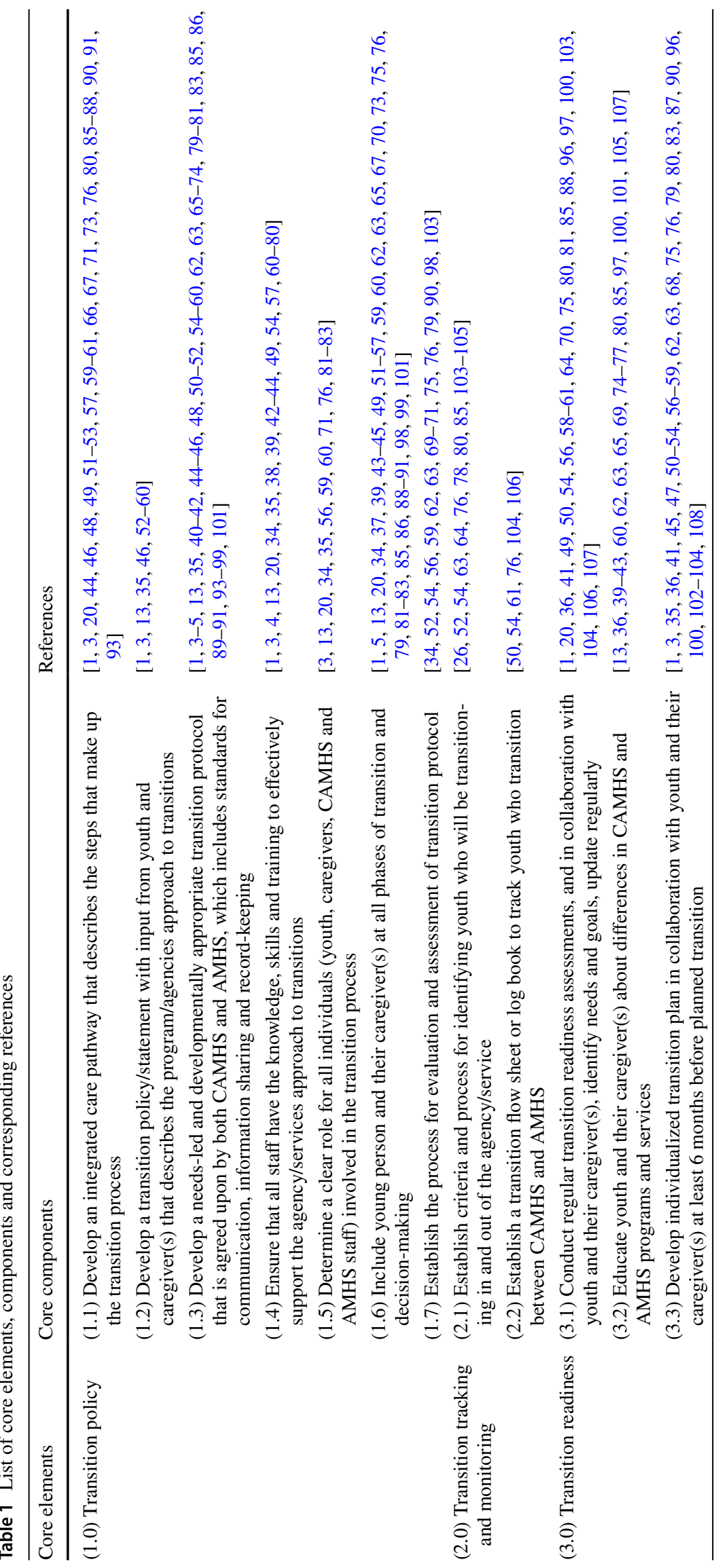




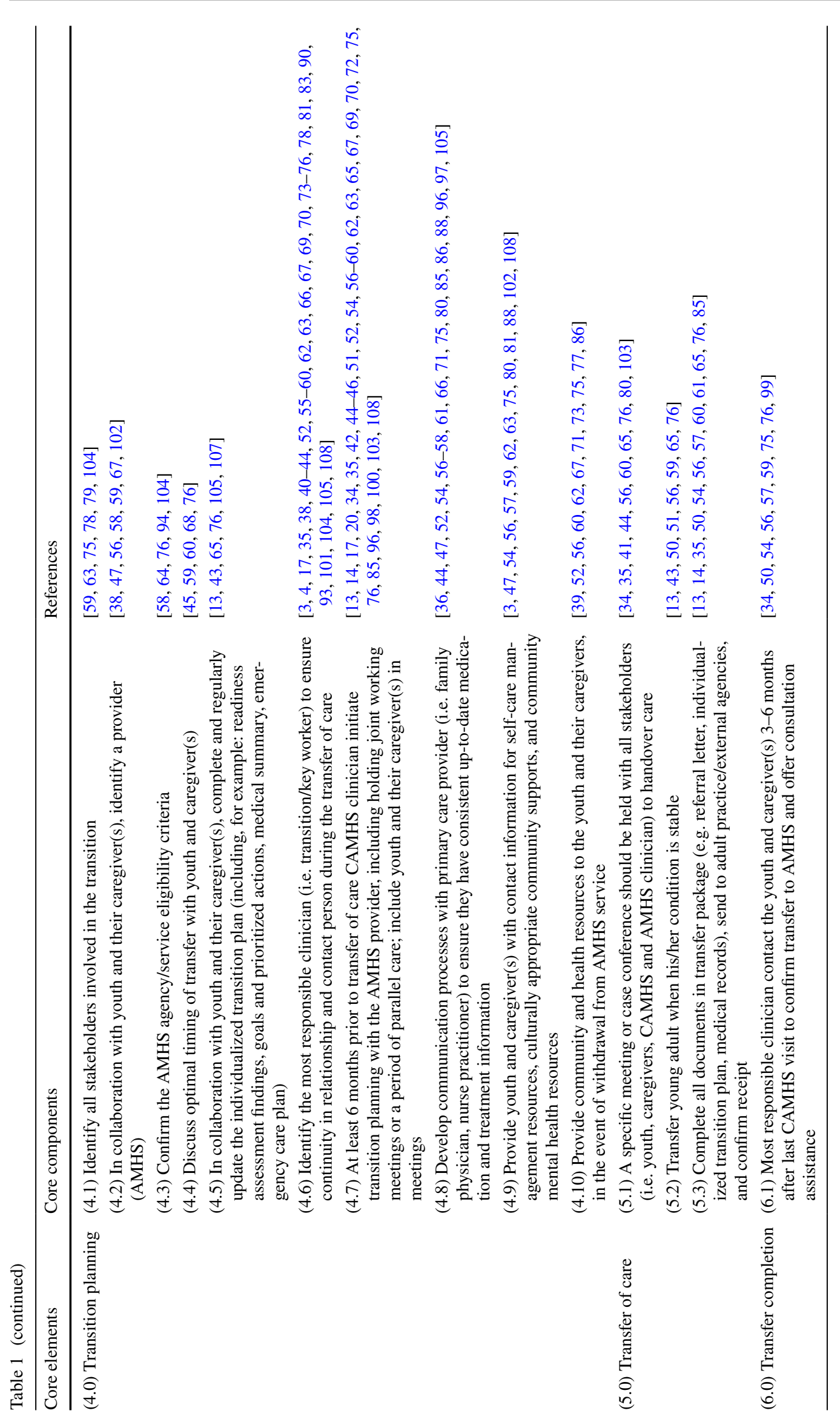


times and to ensure that there is a standard practice for handling waitlists.

\section{Core element 3: transition readiness}

Transition readiness assessments can be used to identify and review the transition needs and goals of youth and their families [20, 36, 41, 49, 50, 54, 56, 58, 59, 64, 70, 75, 80, $81,85,88,96,97,100,103,104,106,107]$. The readiness assessment should review diverse aspects of the youth's life such as physical health, mental health, vocational, housing and educational needs $[59,103]$ as well as the youth's own assessment of their strengths, skills and readiness for transition $[56,75]$. In addition to the transition assessment, an individualized transition plan should be completed in collaboration with youth and their families, rather than by health care professionals alone $[1,3,26,36,41,47,52,54,56-59$, $62,63,68,76,79,83,87,90,102-104,108]$.

The collaborative process of developing goals together with the youth and regularly updating the goals to align with the plan of care was identified as another meaningful contribution to transition $[1,50,54,56,57,60,64,76$, 97]. The development and tailoring of the transition plan to youths' unique needs requires time, with many documents suggesting a minimum of 6 months prior to transition [35, 45, 47, 50-54, 56, 63, 75, 76, 80, 96, 100, 102, 108]. Last, providing youth and their caregiver(s) with information about the differences between the CAMHS and AMHS is another component of transition readiness and was identified in 19 articles [13, 36, 39-42, 60, 62, 63, 69, 74-77, 80, 85, 97, 100, 101]. As noted by Swift et al. [77] education and information sharing may help set expectations and facilitate a feeling of preparedness among youth and their families.

\section{Core element 4: transition planning}

Transition planning was identified as a core element with several associated components. The need to identify all stakeholders from CAMHS, AMHS, pediatric care, the education system and caregivers who will have a role in the transition was identified in several documents $[59,63,75$, $78,79,104]$. Role clarity for all stakeholders is important, particularly when determining which agency takes primary clinical responsibility during the transition process [41, 53, $58,67,76]$. Youth and their caregiver(s), and the CAMHS provider should collaborate in identifying an AMHS provider who will best suit their needs and transition goals [38, $47,56,58,59,67,102]$. The adult care provider will be responsible for supporting the youth after the transition to ensure their needs are being met [47]. This differs from a transition worker or "key worker", who provides continuity throughout the transition process and transfer of care, following the youth as they move from child to adult mental health services. The presence of a key worker was identified as a core component of CAMHS-AMHS transitions in numerous documents $[3,4,17,20,35,38,40-44,52,55-60$, $62,63,66,67,69,70,73-76,78,81,83,90,93,101,104$, $105,108]$.

Several documents recommended that a CAMHS provider initiate transition-related collaboration with an AMHS provider a minimum of 6 months prior to the transfer of care $[44,51,58,59,65,76,103]$. This collaboration could also be the catalyst for a period of parallel care between both agencies $[13,34,44,65,69,70,75,76]$. Five documents identify the significance of transitioning at a time that is most appropriate for the youth $[45,59,60,68,76]$. The youth, their caregiver(s) and the CAMHS provider should agree upon the duration and time of the formal transfer. This might mean extending the period of time in CAMHS if required [68].

It is critical that the young person and their caregiver(s) are engaged in developing and regularly updating the transition plan $[1,34,53,54,56,59,60,70,75,79,85]$. This may include ensuring youth and their families are aware of the services available, know how they can prepare, what it means to transition, and what the transition process will entail $[34,41,45]$. As part of the transition process, youth should be provided resources and tools on strategies to manage their health. Publications recommended providing supports for self-management $[3,54,56,57,59,62,63,75,80$, $81,102]$ as well as contacts for voluntary and community support services [47, 54, 56, 75, 108].

Primary care practitioners, such as a youth's general practitioner (GP or family physician), are critical components of transition planning. Eighteen studies highlighted the importance of ensuring the youth's GP is included in transition and care planning, especially when medications are involved [36, 44, 47, 52, 54, 56-58, 61, 66, 71, 75, 80, $85,86,88,96,105]$.

Five documents $[58,64,76,94,104]$ identified the need to review the eligibility of the AMHS program or service in advance of transition to avoid discontinuity of care should the youth not meet the program or service's criteria. It was suggested in multiple publications that health care providers in both CAMHS and AMHS should take responsibility for ensuring that the youth and their caregivers are made aware of the available mental health and community resources in the event of service gaps, withdrawal from AMHS or if additional support is required beyond the scope of AMHS [39, $52,56,60,62,67,71,73,75,77,86]$.

\section{Core element 5: transfer of care}

Transfer of care involves the logistical components of the discharge and intake processes during the transition [14, 35]. This includes the recommendation that the transfer from CAMHS to AMHS should only occur when the young 
person is stable $[13,43,50,51,56,59,65,76]$. It is recommended that a formal handover of care should occur prior to transfer, include all stakeholders and be made during a multidisciplinary case meeting $[34,35,41,44,56,60,65$, $76,80,103]$. A transfer package should be prepared ahead of this meeting and each stakeholder is given a copy of the package containing the dates of all future appointments, plan of care, goals, pending actions, and legal documents [13, 14, $35,50,54,56,57,60,61,65,76,85]$.

\section{Core element 6: transfer completion}

Transfer completion includes a component that speaks to what needs to occur after the youth has left CAMHS and completed the transfer of care. Several documents recommended that the CAMHS clinicians contact the AMHS service 3-6 months after the last CAMHS visit to confirm the youth has successfully engaged with AMHS and ensure there are no further questions about the referral or transition package $[34,50,54,56,57,59,75,76,99]$.

\section{Discussion}

Our review of the literature identified 56 articles and 30 gray literature eligible documents in an effort to: (1) define CAMHS-AMHS transition success; and (2) identify core components and corresponding transitions indicators that are being applied within the field. Two definitions of child-toadult transition success were dominant among the reviewed documents. One was not specific to mental health [15] while the other was exclusive to CAMHS-AMHS transitions [76]. The latter was based on a recommendation of four criteria hypothesized to lead to CAMHS-AMHS transition success. This definition, however, did not include information on the implementation and measurement of these criteria, which raises issues about variability on how they are interpreted and applied. Although there is a lack of empirical evidence on the use of these criteria, the Singh [76] definition is widely applied in the CAMHS-AMHS transition literature. This lack of empirical evidence reflects the scarcity of intervention studies within the field, a conclusion supported by the systematic review from Embrett et al. [20] of the effectiveness of services and programs for youth transitioning from CAMHS to AMHS.

Our review delineated common core elements of transitions that multiple countries, organizations and disciplines agree upon. These core elements align with those of physical healthcare transitions [12], but have components that are unique to mental healthcare transitions. For example, the Got Transitions transition policy includes several components that emphasize the value of a shared view of how transitions should proceed with input from clinicians, youth, and their families [12]. The mental health literature takes this concept further, emphasizing that youth and their caregiver(s) should be included in decision-making at all phases of transition. Engaging in a shared planning process can promote common understanding between those who provide care (service providers and sectors) and those who receive care (youth and their families) [1, 51, 80].

Several core components of a successful transition were identified in this scoping review that was specific to the roles and responsibilities of the organization and service provider. However, it is important to acknowledge the broader context of system-level issues in mental healthcare systems that may constrain their effective implementation. CAMHS and AMHS are two separate systems with unique funding structures, service philosophies and target populations $[1,37,58$, 109]. Government funding allocations often dictate rigid age cut-offs, where service with CAMHS often ends at age 18 regardless of whether the youth is developmentally ready or connected to AMHS [1, 5, 72, 104]. This can result in service gaps while youth are placed on a waitlist for AMHS. Youth may also experience an eligibility criteria mismatch, where they find that upon transition out of CAMHS they are ineligible for service with AMHS because they do not meet diagnostic criteria $[1,4]$. These issues have significant implications for the feasibility of the implementation of the core components. The close communication between CAMHS and AMHS that would be required to implement these core components would be severely constrained by service gaps and wait times. Even in an ideal scenario where AMHS is prepared to accept a young person directly following their transfer out of CAMHS, separate funding streams complicate the logistics of how providers would bill for collaborative efforts such as joint working meetings, case conferences, or parallel care $[13,104]$. There is considerable debate within the literature about how to address these systemic problems, with options suggested including permitting service continuation from CAMHS based on developmental readiness, specialized services for 16-24-year-olds, expansion of AMHS eligibility criteria, and extending age cut-off for CAMHS to 25; however, no consensus has been reached [4, 67, 68, 83, 102, 104].

At the organizational level, the core components require further detail and clarity to be effectively operationalized. The literature does not clarify or suggest who should take ultimate responsibility for the enactment of the core components. Core components in core elements 1 and 2, for example, the development of policies, protocols and tracking mechanisms, suggest the involvement of organizational leadership. Meanwhile, the other core components implicitly point at clinicians. While collaboration with AMHS is encouraged, CAMHS clinicians seem to bear primary responsibility for the core components of transition readiness, transition planning, transfer of care and transfer 
completion. This raises the question of whether and how tasks such as conducting readiness assessments, developing transition plans, and compiling community resources can be integrated into clinicians' existing workload in a manageable way, or if they require additional funding and resources [13]. Another key question centers around the appropriate involvement of family members and caregivers in the transition process. Although collaboration with family members and caregivers is interwoven throughout the core components, late adolescence is acknowledged as a period where youth move towards increased independence and autonomy from family $[3,53,54]$. The literature stresses that collaboration with youth and their family members needs to be negotiated with sensitivity [3,13, 81], particularly in light of youth's legal rights to confidentiality and increasing responsibility for self-management of their condition [53, 91].

Another important finding of this scoping review is the significant lack of measurable indicators and evaluative tools needed to assess the effectiveness of core components of transitions. Quality and process indicators are critical to the ongoing assessment, monitoring and improvement of mental health care. Evidence-based indicators also permit common reporting of performance measures across hospital and community mental health settings, and the identification of possible causes of discontinuities in care [110]. As such, future research should focus on the development, testing, and refinement of quality and process indicators to assess the performance of hospitals and community agencies in the transitions of youth between mental health services.

Despite the lack of indicators, several documents recommended a post-transition evaluation. However, none offered a framework or tool that would successfully achieve this and implementing this evaluation may be impeded by the lack of transition interventions and critical details on how to operationalize many of the core components. For example, several documents discuss the need for a key worker, yet there is little information about how to identify that individual, how to implement the role, and how to evaluate the role. A further example is the literature that recommends only transitioning the youth from CAMHS to AMHS when their condition is stable. However, there is little in the way of description about what 'stable' or 'stability' means for youth with mental illness and whether stability is realistic or necessary for the transfer of care. These two examples from this review illustrate the need to move beyond identifying and discussing youth and caregiver(s) needs for CAMHS to AMHS transitions to instead conducting research on the development, implementation and evaluation of promising new approaches to improve quality of transitions, and youth and caregiver(s) outcomes.

While our scoping review summarizes current knowledge about the core components of CAMHS-AMHS transition programs and services, it more importantly points to the need for empirical research on the impact and experiences associated with CAMHS-AMHS transitions. Conducting such research will require the development, implementation and evaluation of the quality and process indicators derived from the 26 core components identified in this review.

\section{Future clinical research directions}

Moving forward, consensus on the importance and feasibility of the 26 core components identified in this scoping review is required. Employing a structured Delphi Study $[111,112]$ approach that includes youth, their caregivers, and clinicians can be used to achieve consensus. Such an exercise can provide the evidence needed to select core components and design interventions around them that will facilitate transition success for youth. To our knowledge, no Delphi or group consensus studies have been conducted with experts, including youth, parents, clinicians and administrators, to investigate which transition program components both enhance the transition experience and ensure continuity of care.

Another future clinical and research opportunity involves the development and evaluation of integrated clinical pathway (ICPs) [90]. These ICPs can be informed by the core elements and components identified in this scoping review and co-created in collaboration with youth, families and CAMHS and AMHS providers. ICPs tell the multidisciplinary team, the youth, and their caregiver(s) the steps to be undertaken to facilitate effective transitions and what to expect when youth transition [92, 113, 114]. Importantly, ICPs can provide services (including clinicians) with the information needed to evaluate planned care against what was actually delivered [92], and thus determine the effectiveness of a transition intervention or program. One recent publication, the National Institute for Health and Care Excellence (NICE) guideline "Transition from children's to adults' services for young people using health or social care services", provides practical recommendations to improve the planning and quality of health care transitions. Although it is not specific to mental health care, it offers a model for programs aiming to improve the transition process and experience [56].

Another important focus for future research is the examination of funding structures and fees to understand how they can influence the CAMHS-AMHS transition process. There needs to be a thorough investigation of how mental health care funding and insurance programs at all levels of the mental health care system can impact on the success of transitions between mental health care services.

As this review focused specifically on CAMHS-AMHS transitions, we excluded any studies that describe the experiences of youth who do not transition directly into a service 
or program at AMHS (i.e. youth who return to their GP's for mental health care). There is a need to better understand the needs of this group of youth-including their preferences for ongoing care. There is also a need to pay more attention to the roles and responsibilities of other resources and services, such as GP's, post-secondary education institutions, and community mental health services, among others. The responsibilities of these organizations and services are vaguely defined at present, and it is unclear how they could be (or if they should be) incorporated into the transition process. There is also a need to clearly define the role of models of care that are focused specifically on providing mental health care to transitional aged youth (ages 15-24) in the CAMHS-AMHS transition process need to be clearly defined. This could be achieved by examining the needs of youth during this developmental period, and whether models of care promote continuity of care or provide another transition point that young adults and their caregivers must learn to navigate.

\section{Limitations}

This scoping review has several limitations. First, the literature search may have unintentionally excluded some relevant documents, as it was limited to English language articles and documents. The use of English language only papers may have precluded the understanding of the depth of CAMHS to AMHS in a global context. The majority of the documents reviewed were from North American and European countries which may have limited our understanding of the nature of the issues in other countries. Next, while the search terms "transition to adult care" and "continuity of patient care" are Medical Subject Heading (MeSH) terms, 'transfer' and 'transition' are not. Last, the addition of these MeSH terms is recent; for example "transition to adult care" was added in 2011. In an effort to minimize the effect of these limitations, we screened the reference lists of all included articles to ensure that our literature search strategy did not miss any seminal documents. Last, this review focuses solely on typically developing youth with a primary diagnosis of mental illness. Future studies that focus on transitions among youth with neurodevelopmental conditions (i.e. autism) as a primary diagnosis should be conducted. This population might require specialty services outside the scope of mental illness that could lead to unique transition challenges. It merits a review solely based on those conditions.

\section{Conclusion}

Our scoping review and the associated comprehensive list of core elements and components of CAMHS-AMHS transitions clearly demonstrate that it is possible to develop an integrated pathway and care coordination to improve transition experiences and outcomes. Such improvements would include better quality care, and care pathways that youth themselves have participated in developing, thus reducing the likelihood of youth 'falling through the cracks' as they move from child to adult mental health care. We also promote the engagement of youth and families as central decision-makers, an essential element of successful transitions in mental health care. Our scoping review clearly shows that there are important concepts and measures that can act as catalysts for change in current transition practices.

Acknowledgements Kristin Cleverley was supported by the CAMH Chair in Mental Health Nursing Research while writing this article. The authors wish to thank Alexis Siren, Katye Stevens, and Maureen Rice for their support on this manuscript. This research received no specific grant from any funding agency in the public, commercial, or not-for-profit sectors.

\section{Compliance with ethical standards}

Conflict of interest On behalf of all authors, the corresponding author states that there is no conflict of interest.

Open Access This article is distributed under the terms of the Creative Commons Attribution 4.0 International License (http://creativeco mmons.org/licenses/by/4.0/), which permits unrestricted use, distribution, and reproduction in any medium, provided you give appropriate credit to the original author(s) and the source, provide a link to the Creative Commons license, and indicate if changes were made.

\section{References}

1. Hoffman C, Heflinger CA, Athay M, Davis M (2009) Policy, funding, and sustainability: issues and recommendations for promoting effective transition systems. In: Clark HB, Unruh DK (eds) Transition of youth and young adults with emotional or behavioral difficulties: an evidence-based handbook. Paul $\mathrm{H}$ Brookes Publishing, Baltimore, pp 263-290

2. Ontario Ministry of Health and Long-Term Care (2016) Provincial and territorial health ministers focus on strengthening health care for all Canadians. In: CISION. https://www.newswire.ca/ news-releases/provincial-and-territorial-health-ministers-focus -on-strengthening-health-care-for-all-canadians-597368041. html. Accessed 5 May 2017

3. Davidson S, Cappelli M (2011) We've got growing up to do: transitioning youth from child and adolescent mental health services to adult mental health services. Ontario Centre of Excellence for Child and Youth Mental Health, Ottawa

4. Lamb C, Murphy M (2013) The divide between child and adult mental health services: points for debate. Br J Psychiatry 202:s41-s44. https://doi.org/10.1192/bjp.bp.112.119206 
5. Reale L, Frassica S, Gollner A, Bonati M (2015) Transition to adult mental health services for young people with attention deficit hyperactivity disorder in Italy: parents' and clinicians' experiences. Postgrad Med 127:671-676. https://doi. org/10.1080/00325481.2015.1070658

6. Singh SP, Evans N, Sireling L (2005) Mind the gap: the interface between child and adult mental health services. Psychiatr Bull 29:292-294. https://doi.org/10.1192/pb.29.8.292

7. Stagi P, Galeotti S, Mimmi S et al (2015) Continuity of care from child and adolescent to adult mental health services: evidence from a regional survey in Northern Italy. Eur Child Adolesc Psychiatry 24:1535-1541. https://doi.org/10.1007/s0078 7-015-0735-z

8. Wilson M (2016) Health Accord must address growing mental health crisis in Canada. In: The globe and mail. https:// www.theglobeandmail.com/opinion/health-accord-must-addre ss-growing-mental-health-crisis-in-canada/article32339126/. Accessed 26 May 2017

9. Signorini G, Singh SP, Boricevic-Marsanic V et al (2018) The interface between child/adolescent and adult mental health services: results from a European 28-country survey. Eur Child Adolesc Psychiatry 27:501-511

10. Hofstra MB, Van der Ende J, Verhulst FC (2000) Continuity and change of psychopathology from childhood into adulthood: a 14-year follow-up study. J Am Acad Child Adolesc Psychiatry 39:850-858. https://doi.org/10.1097/00004583-20000 7000-00013

11. Islam Z, Ford T, Kramer T et al (2016) Mind how you cross the gap! Outcomes for young people who failed to make the transition from child to adult services: the TRACK study. B J Psychiatr Bull 40:142-148. https://doi.org/10.1192/pb.bp.115.05069 0

12. McManus M, White P, Prior M et al (2014) Six core elements of health care transition 2.0 transitioning youth to an adult health care provider for use by pediatric, family medicine, and med-peds providers. The National Alliance to Advance Adolescent Health, Washington

13. Singh SP, Paul M, Ford T et al (2010) Process, outcome and experience of transition from child to adult mental healthcare: multiperspective study. Br J Psychiatry 197:305-312. https:// doi.org/10.1192/bjp.bp.109.075135

14. Paul M, Ford T, Kramer T et al (2013) Transfers and transitions between child and adult mental health services. Br J Psychiatry 202:s36-s40. https://doi.org/10.1192/bjp.bp.112.119198

15. Blum RWM, Garell D, Hodgman CH et al (1993) Transition from child-centered to adult health-care systems for adolescents with chronic conditions. J Adolesc Health 14:570-576. https://doi.org/10.1016/1054-139x(93)90143-d

16. Reid R, Haggerty J, McKendry R (2002) Defusing the confusion: concepts and measures of continuity of health care: final report. Canadian Health Services Research Foundation, Ottawa

17. Hovish K, Weaver T, Islam Z et al (2012) Transition experiences of mental health service users, parents, and professionals in the United Kingdom: a qualitative study. Psychiatr Rehabil J 35:251-257. https://doi.org/10.2975/35.3.2012.251.257

18. Riosa PB, Preyde M, Porto ML (2015) Transitioning to adult mental health services: perceptions of adolescents with emotional and behavioral problems. J Adolesc Res 30:446-476. https://doi.org/10.1177/0743558415569730

19. Pottick KJ, Bilder S, Stoep AV et al (2007) US patterns of mental health service utilization for transition-age youth and young adults. J Behav Health Serv Res 35:373-389. https:// doi.org/10.1007/s11414-007-9080-4

20. Embrett MG, Randall GE, Longo CJ et al (2016) Effectiveness of health system services and programs for youth to adult transitions in mental health care: a systematic review of academic literature. Adm Policy Ment Health Ment Health Serv Res 43:259-269. https://doi.org/10.1007/s10488-015-0638-9

21. Di Rezze B, Nguyen T, Mulvale G et al (2015) A scoping review of evaluated interventions addressing developmental transitions for youth with mental health disorders. Child Care Health Dev 42:176-187. https://doi.org/10.1111/cch.12306

22. Fixsen DL, Naoom SF, Blase KA et al (2005) Implementation research: a synthesis of the literature. University of South Florida, Tampa

23. Mainz J (2003) Defining and classifying clinical indicators for quality improvement. Int J Qual Health Care 15:523-530. https ://doi.org/10.1093/intqhe/mzg081

24. Fletcher-Johnston M, Marshall SK, Straatman L (2011) Healthcare transitions for adolescents with chronic life-threatening conditions using a Delphi method to identify research priorities for clinicians and academics in Canada. Child Care Health Dev 37:875-882. https://doi.org/10.1111/j.1365-2214.2011.01318 .$x$

25. Mental Health Commission of Canada (2015) Taking the next step forward: building a responsive mental health and addictions systems for emerging adults. In: Mental Health Commission of Canada. http://www.mentalhealthcommission.ca/English/artic le/77031/September-17-2015-mental-health-commission-canad a-releases-comprehensive-report-suppor. Accessed 15 May 2017

26. NHS England/Medical Directorate/Parity of Esteem Programme (2015) Model specification for transitions from child and adolescent mental health services. NHS England, London

27. Office of the Auditor General of Ontario (2016) Child and youth mental health. In: 2016 annual report of the Office of the Auditor General of Ontario, Ontario, pp 111-147

28. Arksey H, O'Malley L (2005) Scoping studies: towards a methodological framework. Int J Soc Res Methodol 8:19-32. https:// doi.org/10.1080/1364557032000119616

29. Levac D, Colquhoun H, O'Brien KK (2010) Scoping studies: advancing the methodology. Implement Sci 5:1-9. https://doi. org/10.1186/1748-5908-5-69

30. Cleverley K, Bennett K, Jeffs L (2016) Identifying process and outcome indicators of successful transitions from child to adult mental health services: protocol for a scoping review. BMJ Open 6:e012376. https://doi.org/10.1136/bmjopen-2016-012376

31. Canadian Agency for Drugs and Technologies (2015) Grey matters: a practical tool for searching health-related grey literature. Canadian Agency for Drugs and Technologies, Ottawa

32. Landis JR, Koch GG (1977) The measurement of observer agreement for categorical data. Biometrics 33:159-174. https://doi. org/10.2307/2529310

33. Hsieh H-F, Shannon SE (2005) Three approaches to qualitative content analysis. Qual Health Res 15:1277-1288. https://doi. org/10.1177/1049732305276687

34. Lindgren E, Soderberg S, Skar L (2013) The gap in transition between child and adolescent psychiatry and general adult psychiatry. J Child Adolesc Psychiatr Nurs 26:103-109. https://doi. org/10.1111/jcap.12027

35. McNamara N, McNicholas F, Ford T et al (2014) Transition from child and adolescent to adult mental health services in the Republic of Ireland: an investigation of process and operational practice. Early Interv Psychiatry 8:291-297. https://doi.org/10.1111/ eip. 12073

36. Dimitropoulos G, Tran AF, Agarwal P et al (2013) Challenges in making the transition between pediatric and adult eating disorder programs: a qualitative study from the perspective of service providers. Eat Disord 21:1-15. https://doi.org/10.1080/10640 266.2013.741964

37. Dimitropoulos G, Tran AF, Agarwal P et al (2012) Navigating the transition from pediatric to adult eating disorder programs: 
perspectives of service providers. Int J Eat Disord 45:759-767. https://doi.org/10.1002/eat.22017

38. Geenen SJ, Powers LE, Wayne S (2003) Understanding the role of health care providers during the transition of adolescents with disabilities and special health care needs. J Adolesc Health 32:225-233. https://doi.org/10.1016/s1054-139x\%2802\%29003 96-8

39. McNicholas F, Adamson M, McNamara N et al (2015) Who is in the transition gap? Transition from CAMHS to AMHS in the Republic of Ireland. Ir J Psychol Med 32:61-69. https://doi. org/10.1017/ipm.2015.2

40. Sawicki GS, Whitworth R, Gunn L et al (2011) Receipt of health care transition counseling in the national survey of adult transition and health. Pediatrics 128:e521-e529. https://doi. org/10.1542/peds.2010-3017

41. Dowdney L, Bruce H (2014) Transiting out of child and adolescent mental health services-influences on continuities and discontinuities in mental health care. In: Byrne P, Rosen A (eds) Early intervention in psychiatry: EI of nearly everything for better mental health. Wiley, Chichester

42. Hall CL, Newell K, Taylor J et al (2013) 'Mind the gap'-mapping services for young people with ADHD transitioning from child to adult mental health services. BMC Psychiatry 13:186. https://doi.org/10.1186/1471-244x-13-186

43. Health and Social Care Advisory Service (2006) CAMHS to adult transition. HASCAS tools for transition. A literature review for informed practice, Health and Social Care Advisory Service, Great Britain

44. Lamb C, Hall D, Kelvin R, Van Beinum M (2008) Working at the CAMHS/adult interface: good practice guidance for the provision of psychiatric services to adolescents/young adults. Royal College of Psychiatrists, London

45. Lindgren E, Soderberg S, Skar L (2014) Managing transition with support: experiences of transition from child and adolescent psychiatry to general adult psychiatry narrated by young adults and relatives. Psychiatry J 2014:8. https://doi. org/10.1155/2014/457160

46. Moscoso A, Jovanovic N, Rojnic M (2015) Transition from adolescent to adult mental health services in Europe from the provider's perspective. Lancet Psychiatry 2:779-780. https:// doi.org/10.1016/s2215-0366(15)00359-4

47. Robb A, Findling R (2013) Challenges in the transition of care for adolescents with attention-deficit/hyperactivity disorder. Postgrad Med 125:131-140. https://doi.org/10.3810/ pgm.2013.07.2685

48. Singh SP (2009) Transition of care from child to adult mental health services: the great divide. Curr Opin Psychiatry 22:386390. https://doi.org/10.1097/yco.0b013e32832c9221

49. Systems Improvement through Service Collaboratives (2013) Headspace. Centre for Addiction and Mental Health, Toronto

50. McManus M, White P, Pirtle R et al (2015) Incorporating the six core elements of health care transition into a medicaid managed care plan: lessons learned from a pilot project. J Pediatr Nurs 30:700-713. https://doi.org/10.1016/j.pedn.2015.05.029

51. Arcelus J, Bouman WP, Morgan JF (2008) Treating young people with eating disorders: transition from child mental health to specialist adult eating disorder services. Eur Eat Disord Rev 16:30-36. https://doi.org/10.1002/erv.830

52. Appleton S, Pugh K (2011) Planning mental health services for young adults-improving transition. A resource for health and social care commissioners. National Mental Health Development, London

53. Koroloff NM (1990) Moving out: transition policies for youth with serious emotional disabilities. J Ment Health Adm 17:7886. https://doi.org/10.1007/bf02518582
54. Ministry of Health (2014) Transition planning guidelines for infant, child and adolescent mental health/alcohol and other drugs services. Ministry of Health, Wellington

55. National Children's Bureau (2010) VSS policy briefing: transitions between children's and adult's health services, and the role of voluntary and community children's sector. NCB

56. National Institute for Health and Care Excellence (2016) Transition from children's to adults' services for young people using health or social care services: NICE guideline. National Institute for Health and Care Excellence

57. England NHS (2015) 2014/15 NHS STANDARD CONTRACT: model transfer of and discharge from care protocol for young people with mental health problems in transition. NHS England, London

58. Parker C, Clements L, Harbour A, Honigmann J (2010) Transitions in mental health care-a guide for health and social care professionals on the legal framework for the care, treatment and support of young people with emotional and psychological problems during their transition years. YoungMinds, London

59. Queensland Government Department of Health (2015) Guideline for the transition of care for young people receiving mental health services. Queensland Government, Queensland

60. Royal College of Nursing (2013) Lost in translation-moving young people between child nad adult health services. Royal College of Nursing, London

61. Ayton A, Meads G (2012) The implementation of the Care Programme Approach in the West Midlands CAMHS: the impact of organisational diversity on patient safety - an extended audit. Int J Clin Leadersh 17:185

62. Brodie I, Goldman R, Clapton J (2011) Mental health service transitions for young people. Social Care Institute for Excellence, London

63. City of Brantford (2015) Transitioning youth from children's mental health to adult mental health and addictions services community protocol. Social Services Strategic Planning Department, Norfolk

64. Davis M, Sondheimer DL (2005) State child mental health efforts to support youth in transition to adulthood. J Behav Health Serv Res 32:27-42. https://doi.org/10.1007/bf02287326

65. Government of South Australia (2014) South Australian youth mental health system of care operational guidelines. Government of South Australia, South Australia

66. Hall CL, Newell K, Taylor J et al (2015) Services for young people with attention deficit/hyperactivity disorder transitioning from child to adult mental health services: a national survey of mental health trusts in England. J Psychopharmacol 29:39-42. https://doi.org/10.1177/0269881114550353

67. Lamont E, Harland J, Atkinson M, White R (2009) Provision of mental health services for care leavers: transition to adult services (LGA research report). Local Government Association, Slough

68. Mandarino K (2014) Transitional-age youths: barriers to accessing adult mental health services and the changing definition of adolescence. J Hum Behav Soc Environ 24:462-474. https://doi. org/10.1080/10911359.2013.835760

69. McLaren S, Belling R, Paul M et al (2013) "Talking a different language": an exploration of the influence of organizational cultures and working practices on transition from child to adult mental health services. BMC Health Serv Res 13:254. https:// doi.org/10.1186/1472-6963-13-254

70. Muñoz-Solomando A, Townley M, Williams R (2010) Improving transitions for young people who move from child and adolescent mental health services to mental health services for adults: lessons from research and young people's and practitioners' experiences. Curr Opin Psychiatry 23:311-317. https://doi. org/10.1097/yco.0b013e32833a51e2 
71. Paul M, Street C, Wheeler N, Singh SP (2015) Transition to adult services for young people with mental health needs: a systematic review. Clin Child Psychol Psychiatry 20:436-457. https://doi. org/10.1177/1359104514526603

72. Reale L, Bonati M (2015) Mental disorders and transition to adult mental health services: a scoping review. Eur Psychiatry 30:932-942. https://doi.org/10.1016/j.eurpsy.2015.07.011

73. Rethink (2011) Joint working at the interface - early intervention in psychosis and specialist child and adolescent mental health services. Rethink, London

74. Richards M, Vostanis P (2004) Interprofessional perspectives on transitional mental health services for young people aged 16-19 years. J Interprof Care 18:115-128. https://doi. org/10.1080/13561820410001686882

75. Sainsbury M, Goldman R (2011) Mental health service transitions for young people. Social Care Institute of Excellence, London

76. Singh SP, Paul M, Islam Z et al (2010) Transition from CAMHS to adult mental health services (TRACK): a study of policies, process and user and carer perspective (TRACK). Queen's Printer and Controller of HMSO 2010

77. Swift KD, Hall CL, Marimuttu V et al (2013) Transition to adult mental health services for young people with attention deficit/hyperactivity disorder (ADHD): a qualitative analysis of their experiences. BMC Psychiatry 13:1-11. https://doi. org/10.1186/1471-244x-13-74

78. Systems Improvement through Service Collaboratives (n.d.) Ottawa Transitional Youth Pilot Project. Centre for Addiction and Mental Health. http://eenet.ca/sites/default/files/pdfs/Ottaw a-Transitional-Youth-Pilot-Project.pdf

79. Van Rensburg K (2009) Transition services. Clinical psychology in practice. BPS Blackwell, Chichester, pp 288-295

80. Young S, Murphy CM, Coghill D (2011) Avoiding the "twilight zone": recommendations for the transition of services from adolescence to adulthood for young people with ADHD. BMC Psychiatry 11:174. https://doi.org/10.1186/1471-244x-11-174

81. Sawin KJ, Cox AW, Metzger SG et al (1999) Transition planning for youth with chronic conditions: an interdisciplinary process. Natl Acad Pract Forum 1:183-196

82. Singh SP, Paul M, Ford T et al (2008) Transitions of care from child and adolescent mental health services to adult mental health services (TRACK study): a study of protocols in Greater London. BMC Health Serv Res 8:1-7. https://doi. org/10.1186/1472-6963-8-135

83. Vloet MA, Davidson S, Cappelli M (2011) "We suffer from being lost": formulating policies to reclaim youth in mental health transitions. Healthc Q 14:32-38. https://doi.org/10.12927 /hcq.2011.22361

84. Belling R, Whittock M, McLaren S et al (2011) Achieving continuity of care: facilitators and barriers in community mental health teams. Implement Sci IS 6:23. https://doi. org/10.1186/1748-5908-6-23

85. Boilson M, Forbes F, Quilter M, Sutherland C (2013) Attention deficit hyperactivity disorder (ADHD) guidance for transition from child and adolescent services to adult services. Royal College of Psychiatrists, London

86. Health and Social Care Scrutiny Commission (2013) Mental health transition (16-24 years) - a report from the Health and Social Care Scrutiny Commission. Health and Social Care Scrutiny Commission

87. Ogundele MO (2013) Transitional care to adult ADHD services in a North West England district. Clin Gov 18:210-219. https:// doi.org/10.1108/cgij-01-2013-0001

88. Pitts M (2014) Attention deficit hyperactivity disorder: transition to adult care. Nurse Prescr 12:345-348. https://doi.org/10.12968 /npre.2014.12.7.345
89. Provincial System Support Program (2012) SISC Evidence Brief \#1: effective transitions in child and youth mental health and addiction services: protocols, Pathways and Partnerships. Centre for Addiction and Mental Health, Toronto

90. Ubido J, Scott-Samuel A (2015) Rapid evidence review series: effective pathway from child to adult mental health services. Liverpool Public Health Observatory, Liverpool

91. Yarrow D, O'Connor M, Cooper J et al (2008) Making Hillingdon fit for the future-report of the transition from child to adult mental health services working group. Hillingdon, London

92. Campbell H, HotchKiss R, Bradshaw N, Porteous M (1998) Integrated care pathways. BMJ 316:133-137. https://doi. org/10.1136/bmj.316.7125.133

93. Belling R, McLaren S, Paul M et al (2014) The effect of organisational resources and eligibility issues on transition from child and adolescent to adult mental health services. J Health Serv Res Policy 19:169-176. https://doi.org/10.1177/1355819614527439

94. Bruce H, Evans N (2008) The transition into adult care. Psychiatry 7:399-402. https://doi.org/10.1016/j.mppsy.2008.07.001

95. Unruh DK, Waintrup M, Canter T, Smith S (2009) Special service systems for serving youth in the juvenile justice system. In: Clark HB, Unruh DK (eds) Transition to adulthood for young people with emotional and/or behavioral difficulties: an evidenced-based handbook. Paul H Brookes Publishing, Baltimore, pp 189-208

96. Coghill D (2015) Services for adults with ADHD: work in progress. Psychiatr Bull 39:140-143. https://doi.org/10.1192/ pb.bp.114.048850

97. Dimitropoulos G, Toulany A, Herschman J et al (2015) A qualitative study on the experiences of young adults with eating disorders transferring from pediatric to adult care. Eat Disord 23:144-162. https://doi.org/10.1080/10640266.2014.976106

98. Joint Commissioning Panel for Mental Health (2012) Guidance for commissioners of mental health services for young people making the transition from child and adolescent to adult services. Practical mental healthcommissioning, vol 2. Joint Commissioning Panel for Mental Health

99. McMillan I (2011) Bridging the gap between children and adult services. Ment Health Pract 15:6-7

100. Reale L, Costantino MA, Sequi M, Bonati M (2014) Transition to adult mental health services for young people with ADHD. J Atten Disord 22:601-608. https://doi.org/10.1177/1087054714 560823

101. McGrandles A, McMahon K (2012) Transition from child and adolescent to adult mental health services. Br J Nurs 21:10311039. https://doi.org/10.12968/bjon.2012.21.17.1031

102. Singh SP, Tuomainen H (2015) Transition from child to adult mental health services: needs, barriers, experiences and new models of care. World Psychiatry 14:358-361. https://doi. org/10.1002/wps.20266

103. Moosa F, Sandhu T (2015) Transition from children's to adult services for patients with ADHD: a model of care. Adolesc Psychiatry 5:22-30

104. Cappelli M, Davidson S, Racek J et al (2016) Transitioning youth into adult mental health and addiction services: an outcomes evaluation of the youth transition project. J Behav Health Serv Res 43:597-610. https://doi.org/10.1007/s11414-014-9440-9

105. Care Quality Commission (2014) From the pond into the seachildren's transition to adult health services. Care Quality Commission, Gallowgate

106. McManus M, Pollack L, Cooley C et al (2013) Current status of transition preparation among youth with special needs in the United States. Pediatrics 131:1090-1097. https://doi. org/10.1542/peds.2012-3050

107. Holmes J, Loh E (2015) A clinical audit of transitional care in adolescents with ADHD at Blakesley Centre CAMHS. 
ADHD Attent Deficit Hyperact Disord 7:S58-S59. https://doi. org/10.1007/s12402-015-0169-y

108. Oxfordshire and Buckinghamshire Mental Health NHS AUDIT of transition protocol-CAMHS to adult services. NHS Foundation Trust

109. Signorini G, Singh SP, Boricevic-Marsanic V et al (2017) Architecture and functioning of child and adolescent mental health services: a 28-country survey in Europe. Lancet Psychiatry 4:715-724. https://doi.org/10.1016/s2215-0366(17)30127-x

110. Farquhar M (2008) AHRQ quality indicators. In: Hughes R (ed) Patient safety and quality: an evidence-based handbook for nurses. Agency for Healthcare Research and Quality (US), Rockville
111. Hasson F, Keeney S, McKenna H (2000) Research guidelines for the Delphi survey technique. J Adv Nurs 32:1008-1015. https:// doi.org/10.1046/j.1365-2648.2000.t01-1-01567.x

112. Jorm AF (2015) Using the Delphi expert consensus method in mental health research. Aust N Z J Psychiatry 49:887-897. https ://doi.org/10.1177/0004867415600891

113. Peet M, Wakefield S (2002) Integrated Care Pathways in Mental Health: the Need for the 'Human Touch'. J Integr Care Pathw 6:108-114. https://doi.org/10.1177/147322970200600303

114. National Health Service (2011) Integrated care pathways for child and adolescent mental health. In: Integrated care pathways for mental health. http://www.icptoolkit.org/child_and_adole scent_pathways.aspx. Accessed 15 May 2017 